\title{
(2) OPEN ACCESS \\ Evaluating smoking control policies in the e-cigarette era: a modelling study
}

\author{
Thi Thanh Tra Doan 지 , Ken Wei Tan, Borame Sue Lee Dickens, Yin Ai Lean, \\ Qianyu Yang, Alex R Cook
}

- Additional material is published online only. To view please visit the journal online (http://dx.doi.org/10.1136/ tobaccocontrol-2019-054951).

Saw Swee Hock School of Public Health, National University of Singapore and National University Health System, Singapore

\section{Correspondence to}

Alex R Cook, Saw Swee Hock School of Public Health, National University of Singapore, 119077, Singapore; ephcar@nus.edu.sg

TTTD and KWT contributed equally.

Received 17 January 2019 Revised 30 July 2019 Accepted 7 August 2019 Published Online First 4 September 2019
Check for updates

(c) Author(s) (or their employer(s)) 2020. Re-use permitted under CC BY-NC. No commercial re-use. See rights and permissions. Published by BMJ.

To cite: Doan TTT, Tan KW, Dickens BSL, et al.

Tob Control

2020;29:522-530.

\section{ABSTRACT}

Background In jurisdictions in which electronic cigarettes are currently prohibited, policy makers must weigh the potentially lower risk compared with conventional cigarettes against the risk of initiation of e-cigarettes among non-smokers.

Methods We simulated a synthetic population over a 50-year time horizon with an open cohort model using data from Singapore, a country where e-cigarettes are currently prohibited, and data from the USA, the UK and Japan. Using the smoking prevalence and the qualityadjusted life year gained calculated, we compared tobacco control policies without e-cigarettes - namely, raising the minimum legal age (MLA), introducing a smoke-free generation (SFG) and tax rises on tobacco consumption — with policies legalising e-cigarettes, either taking a laissez-faire approach or under some form of restriction. We also evaluated combinations of these policies.

Results Regardless of the country informing the transition probabilities to and from e-cigarette use in Singapore, a laissez-faire e-cigarette policy could reduce the smoking prevalence in the short term, but it is not as effective as other policies in the long term. The most effective single policies evaluated were SFG and aggressive tax rises; the most effective combination of policies considered was MLA plus moderate tax rises and e-cigarettes on prescription.

Conclusion Policy makers in jurisdictions in which ecigarettes are not yet established may be advised not to prioritise e-cigarettes in their tobacco end-game strategy, unless their use can be restricted to current smokers seeking to quit.

\section{INTRODUCTION}

Since their development in the early years of the 21st century, electronic cigarettes (e-cigarettes) have grown rapidly in popularity as an alternative to conventional tobacco-based cigarettes. ${ }^{1-5}$ This growth challenges the mainstream regulatory paradigm of increasing controls on smoking, as the public health goals of reducing harm from nicotine may potentially also be achieved by encouraging smokers to switch from conventional to e-cigarettes, either as a replacement or as a cessation tool. ${ }^{6-8} \mathrm{~A}$ major concern for public health policy makers is the gateway effect,when non-smokers first initiate use of e-cigarettes then transition to smoking conventional cigarettes. ${ }^{9}{ }^{10}$ In theory-though this has yet to be observed in practice due to the recent emergence of e-cigarettes-the gateway effect could lead to greater incidence of nicotine use, reversing gains in global tobacco control efforts. Of particular concern is the risk that uptake among youth may exceed use of conventional cigarettes, as e-cigarettes become normalised. ${ }^{14}$

As a result of the tension between the possible drawbacks and benefits of widespread e-cigarette use, different jurisdictions have taken opposing approaches to regulating the sale or use of e-cigarettes, the penetration of which into different markets differs accordingly. In the UK, for instance, in 2017 , about $4.7 \%$ of people above 15 years old were current e-cigarette users, while in Italy the prevalence of current e-cigarette users is only about $0.2 \%{ }^{11}$ In Asia, some countries have adopted e-cigarettes, such as Philippines ${ }^{12}$ and South Korea, ${ }^{13}$ while Japan has banned e-cigarettes with nicotine ${ }^{14}$ (though not regulated e-cigarettes otherwise) and other countries do not allow the sale of e-cigarettes, such as the United Arab Emirates, ${ }^{12}$ Thailand ${ }^{12} 15$ and Singapore. ${ }^{12}$

Singapore has a history of strong anti-tobacco measures, including designating smoke-free public places and transport, prohibiting advertising, promotion and sponsorship as well as enforcing strict rules for packaging and labelling of tobacco products. ${ }^{16}$ Cigarettes are the dominant tobacco product in the country, with menthol cigarettes common as a gateway product. ${ }^{17}$ Products such as e-cigarettes, smokeless cigars, smokeless cigarillos or smokeless cigarettes are prohibited. ${ }^{18}$ The citystate has also recently raised the minimum legal age (MLA) to buy or consume conventional cigarettes to $21 .{ }^{19}$ To discourage smoking in adolescents, as many underage smokers report obtaining cigarettes from older peers, ${ }^{20}{ }^{21}$ Khoo et $a l^{22}$ have proposed a further extension of the MLA policy to create a 'smoke-free generation' (SFG) beginning with the birth cohort of 2000.

Public health policy makers in countries in which e-cigarettes remain controlled, like Singapore, are therefore faced with a quandary: to legalise the use of e-cigarettes with the intention of encouraging users of conventional cigarettes to switch to the putatively lower risk product despite the potential risk of creating a new generation of e-cigarette users, or to continue prohibiting e-cigarettes and to advance towards an endgame for conventional cigarettes. Such comparisons can be undertaken through mathematical modelling, in which a consistent experimental framework is used to field policies against each other in silico.

To evaluate the potential impacts of these policies on population health, we developed a microsimulation model of cigarette and e-cigarette use over a 50-year time horizon in a synthetic population 
representing Singapore. We use this to assess potential prevalence of nicotine use and of quality-adjusted life years (QALYs) gained under various alternative scenarios, including the recent raising of the MLA to 21, the SFG for birth-cohorts 2000 onwards, the legalisation of e-cigarettes, either on prescription or on general sale, and the raising of tax on tobacco consumption, which we compared with a status quo (SQ) scenario.

\section{METHODS}

\section{Open-cohort model}

We developed an open-cohort microsimulation model to assess the potential impact of different policies for tobacco control in Singapore (see figure 1). Open-cohort models are beneficial for modelling smoking policies as they allow for entry of new individuals at each discrete time step, rather than taking a single static population and projecting forward in time, as the latter approach does not take into account the entry of a younger birth cohort for each subsequent year, which may preferentially advantage one policy over another. First, the model was initialised with forecasted numbers of people aged 11 from 2017 to 2067 representing the birth cohort of each year following the population trend for Singapore as estimated in the $\mathrm{R}$ package wpp2017. ${ }^{23}$ For the starting year of 2017, we included the rest of the population aged $12-80$ years. We also allow for a $3 \%$ annual decrease in mortality rates. ${ }^{24}$ Age pyramids are presented in online supplement figure S1-1. Prevalence of cigarette or e-cigarette users and QALYs were then calculated for living people aged 12-80 years in each year from 2018 to 2067.

The core of the model is a Markov chain with five different states for an individual in a given year: never user, cigarette user, ex-smoker, e-cigarette user and dual user. Age-specific transition probabilities between the first three states were estimated for Singapore using local data (prevalence presented in online supplement figure S1-2). As local data on transitions to and from the e-cigarette and dual user states were not available as e-cigarettes are prohibited in Singapore, we supplemented the transition probabilities with external data from three countries in which e-cigarettes are more established. Using three sets of transition probabilities based on data from the US (Population Assessment of Tobacco and Health study, 2014-2016), ${ }^{25}$ the UK (UK Office for National Statistics, 2014-2017) ${ }^{26}$ and Japan (Tabuchi et al, 2015-2017), ${ }^{27}$ we developed model variants, namely, SGUS, SGUK and SGJP, in which the transitions to and from e-cigarette and dual use follow those in the three countries, respectively. The conversion from study-specific definitions to our categories may be found in online supplement 1 . We sought common patterns which emerge despite the differences between countries and surveys to project the possible range of the smoking prevalence and QALYs gained.

From the US data, most of the e-cigarette users below 25 years old are never smokers, while most of the e-cigarette users above 25 years old have used cigarettes before. Hence, for young e-cigarette users, when they quit, we allowed them to return to the never-smoker state, whereas for older e-cigarette users, when they quit, they become ex-smokers (see figure 1).

In the sensitivity analysis, we present the result of a model where all e-cigarette users when quitting return to the neversmoker state (see online supplement S5).

\section{Estimation of transition probabilities}

We obtained the population estimates of transitions for the USA from the longitudinal survey data with R's survey ${ }^{28}$ package (see online supplement 1). To obtain transition probabilities based on the cross-sectional data of Singapore, the UK and Japan, we use Markov Chain Monte Carlo and the rjags ${ }^{29}$ package. After scaling down the transition probabilities of the first three states using Singapore data to fit with the remaining transition probabilities from the three established markets (the USA, the UK and Japan), we obtained the final transition probability matrix for all five states.

The prior distributions for each model's parameters are provided in online supplement 1 , together with other details on the Markov model formulation.

\section{Relative mortality and quality-adjusted life years}

We took 2.8 to be the relative risk of mortality for cigarette smokers who are less than 60 years old. ${ }^{30}$ The excess risk for ex-smokers was assumed to be $5 \%$ that for cigarette users. ${ }^{31} \mathrm{It}$ has been suggested that a plausible range for the excess relative risk for e-cigarette use is 5\%-40\% of the excess risk experienced by cigarette users. ${ }^{31-35}$ Therefore, for the results in the main text, we used an excess risk value of $10 \%$ for SGUS and SGUK variants (on the optimistic end of the range) and of 5\% for SGJP variant, as legally available e-cigarettes in Japan do not contain nicotine. We also performed sensitivity analysis for different values of the excess risk of e-cigarettes (see online supplement S5). The relative risk for dual users was calculated as the geometric mean of the relative risk for cigarette users and e-cigarette users. These relative risks were reduced for those aged over 60 years as relative mortality decreases with age. ${ }^{36}$

In addition, we accounted for the transition of the relative mortality and QALY over time when users transition to a state of lower risk. Specific values of relative risks used and the formulae for transitions are in online supplement S1.

All QALY results in the main text are presented with no future discounting. We also derived QALYs under a 3\% annual discount (see online supplement S4).

To accommodate other uncertainties in some key parameters, we conducted a sensitivity analysis (as described in online supplement S5), varying the mortality effects of e-cigarettes, initiation rates, use of e-cigarettes as a cessation aid and alternative methods of merging rates from USA, UK and Japan with Singapore.

\section{Scenarios}

Twelve policy scenarios were evaluated for their effect on smoking prevalence and annual QALYs gained, together with a baseline scenario reflecting the SQ. The SQ scenario includes all current tobacco control policies in Singapore as of 2017. In this scenario, only the transition probabilities between never user, cigarette user and ex-smoker were used.

In the MLA scenario, the MLA to smoke conventional cigarettes is raised from 18 to 21 years old. This was modelled by setting the transition probabilities of those below 21 years old to the current transition probabilities of those below 18 years old. This policy has now taken effect.

In the SFG scenario, people born in the year 2000 onwards are never allowed to smoke. In the model, the transition probabilities of the people in birth cohort 2000 and later were set to the mean of the current transition probabilities of illegal smoking among under-aged youths or the current transition probabilities, whichever is smaller. We let the rate of illegal initiation of cigarettes decrease by $3 \%$ annually as the supply of cigarettes decreases.

We simulated two tax scenarios, similar to the policy implemented in Australia between 2013 and 2020. ${ }^{37}$ In both, the tax 
OPEN COHORT MODEL

Birth Cohorts: 1937 to 2055

Time Frame: 2017 to 2067

Ages of Interest: 12 to 80 years

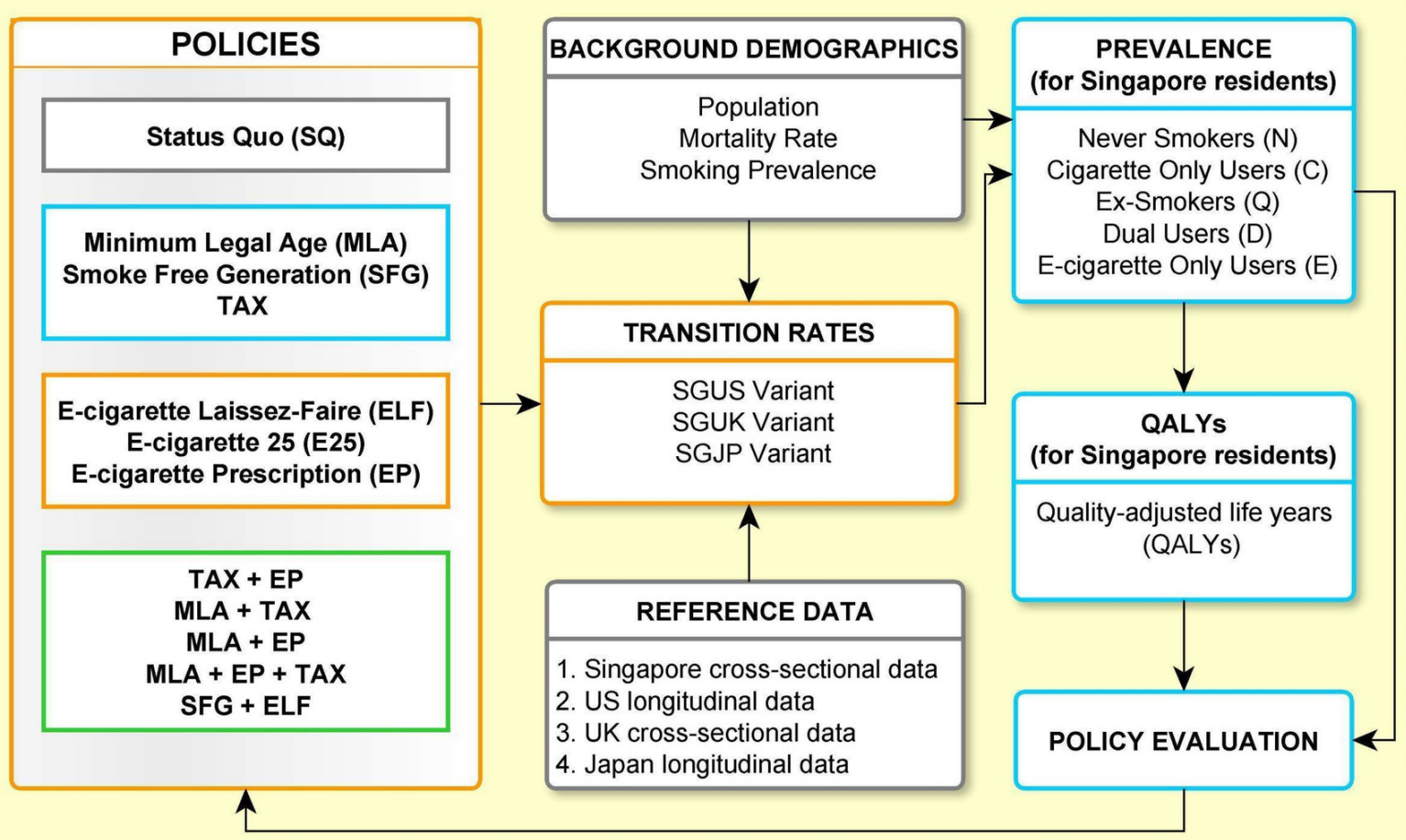

TRANSITIONS BETWEEN STATES FOR YOUTH (12 TO 24 YEARS OLD)

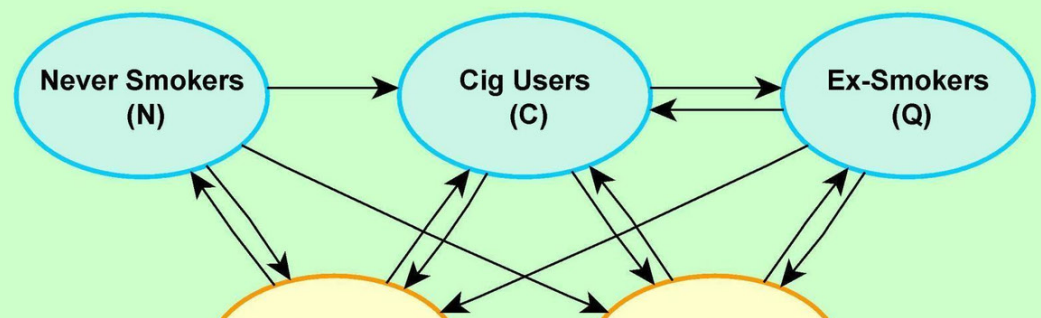

Ecig Users

(E)

Dual Users

(D)

TRANSITIONS BETWEEN STATES FOR ADULTS 25 YEARS OLD AND OLDER

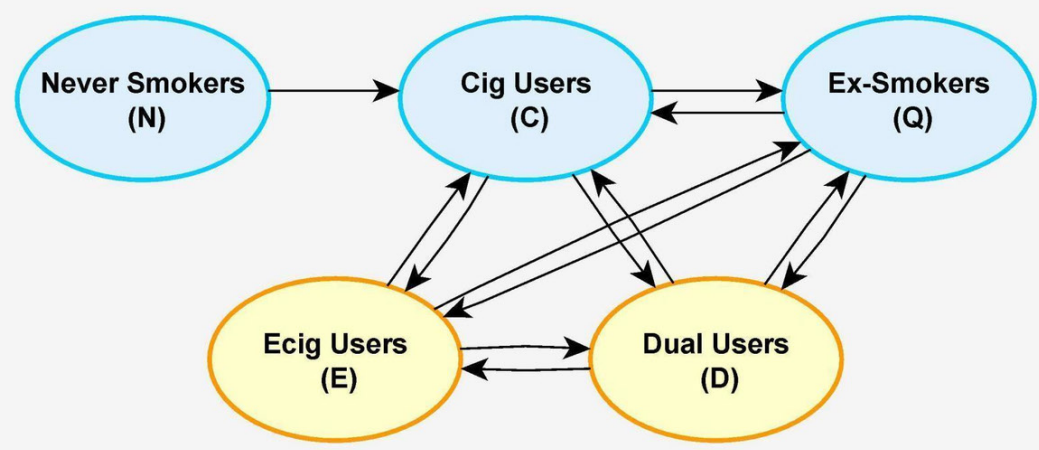

Figure 1 Model structure. 
on tobacco consumption is raised 10 times by $10 \%$ each time relative to the previous price. The tax is raised once every 2 years in the TAX2 scenario, and once every 5 years in the TAX 5 scenario. Based on previous estimates, the price elasticity of smoking prevalence is about -0.2 for developed countries, ${ }^{38}$ that is, a $10 \%$ increase in price is associated with a $2 \%$ relative decline in prevalence. To determine the appropriate change in the initiation rate, quit rate and relapse rate of cigarettes, we used Markov Chain Monte Carlo to estimate the rates that would lead to such a change in prevalence and replaced the model rates accordingly.

The final three scenarios reflect the effects of introducing e-cigarettes into the Singapore market. In the e-cigarette laissezfaire (ELF) scenario, all people aged 18 and above are allowed to buy and use e-cigarettes, whereas in the e-cigarette on prescription (EP) scenario, the sale of e-cigarettes is restricted to cigarette smokers with prescriptions from medical doctors only. To represent this, we set the initiation rate from never smokers to e-cigarette vapers in the EP scenario to zero. For the transitions of current smokers who intend to quit to dual users, to e-cigaretteonly vapers or to ex-smokers, we used the corresponding transition probabilities from a randomised trial in the $\mathrm{UK}^{39}$ in which participants could choose their e-liquid for use in refillable e-cigarettes and were provided face-to-face support to quit.

In the e-cigarette 25 (E25) scenario, only people aged 25 years and above are allowed to buy and use e-cigarettes. The transition probabilities relating to e-cigarettes of people below 25 years old are set to the current transition probabilities of people below 18 years old.

We also simulated the following combinations of policies:

- Raising the MLA and implementing a regime of moderate tax increases (TAX5).

- Raising the MLA and allowing e-cigarettes on prescription (EP).

- Implementing a regime of moderate tax increases (TAX5) and allowing e-cigarettes on prescription (EP).

- All three interventions aforementioned (MLA+TAX5+EP).

- Implementing a SFG policy in tandem with a laissez faire approach to e-cigarettes (ELF).

In the combination of SFG and ELF, we assumed that the e-cigarette initiation rate increases by $1 \%$ annually as the supply of the competing product, cigarettes, diminishes.

The model was built with R V.3.4.4, ${ }^{40}$ and the model structure (figure 1) was drawn with yEd Graph Editor V.3.18.1. ${ }^{41}$

\section{RESULTS}

\section{Transition probabilities}

The US panel data suggest that, as with conventional cigarettes, initiation rates among non-smokers into e-cigarette use are substantial only among those in their teens and 20s, being 3 per 1000 person-years (95\% credible interval (CI) $2 \%$ to $4 \%$ ) for ages 12-17 years and 7 per 1000 person-years (95\% CI 5\%o to 9\%0) for ages 18-24 years. From the data in the UK, the initiation rate was estimated to be 4 per 1000 person-years (95\% CI $0 \%$ to $9 \%$ ) for ages $15-19$ years and 3 per 1000 person-years (95\% CI 0\%o to 8\%o) for ages 20-24 years. In Japan, the overall e-cigarette initiation rate was lower (at 2 per 1000 person-years, $95 \%$ CI $0 \%$ to $7 \%$ ), but after setting the initiation rate for those aged 30 years or more to 0 , the initiation rate for ages 15-29 years would be 8 per 1000 person-years (95\% CI 0\%o to $26 \% 0$ ), that is, comparable to the US-derived estimates. The initiation rate of conventional cigarettes (from non-smokers) was higher in the USA study than that of e-cigarettes for all age groups except those under 18 years old, for whom slightly more transitioned to smoking e-cigarettes than conventional cigarettes. The UK and Japanese data both showed lower initiation of e-cigarettes than conventional cigarettes (estimated rates are presented in online supplement S2).

The US panel data show some potential evidence of a gateway effect, with about 23 transitions per 1000 person-years $(95 \% \mathrm{CI}$ $22 \%$ to $23 \% 0$ ) from e-cigarette use only to conventional cigarette use and 36 per 1000 person-years transitioned (95\% CI $35 \%$ to $37 \%$ ) from e-cigarette use only to dual use in those aged 12-17 years (both presented in online supplement S2). (The UK and Japanese data, being cross-sectional, cannot give reliable estimates of the gateway effect.)

The internal validity of these estimates is shown in the close correspondence between estimated and empirical prevalence in the four settings considered (see online supplement S2). Extrapolating the Singapore estimates of transition rates forward in the SQ scenario suggests that if no new policies are implemented, the prevalence of current smokers will stabilise at about $14 \%$ (95\% CI $13.7 \%$ to $14.4 \%$ ) by 2067 , similar to current rates, despite the substantially changing age profile of the population.

\section{Scenario analyses}

Prevalence of cigarette and e-cigarette use

Regardless of which country informs the transition rates to and from e-cigarette and dual use, the prevalence of tobacco use under ELF and EP reaches a plateau after descending initially (figure 2). Under ELF, the reduction in cigarette prevalence is most notable in the SGUK variant and negligible in the SGUS variant. This reflects the relatively high flux from conventional to e-cigarette use and low initiation rate of e-cigarettes suggested by the UK data (online supplement S2). On the other hand, under EP, the decline in cigarette use is more significant and consistent across the variants as only smokers who intend to quit are allowed to use e-cigarettes.

Among the seven scenarios of single policies considered, an aggressive tax regime (with prices rising 10\% every 2 years over two decades, TAX2) and the SFG have the greatest long-term impact in reducing the prevalence of cigarette use, followed by less intensive policies (MLA and a quinquennial tax rise, TAX5).

\section{Quality-adjusted life years (QALYs) gained compared with status quo (SQ)}

The projected QALYs gained follow a pattern consistent with that of e/cigarette prevalence; figure 3 contains the projection of QALYs and online supplement figure S4-1, the total QALYs gained under each scenario in 2017-2067. Under the policies including e-cigarettes, early annual QALY gains could be realised as cigarette smokers switch to e-cigarette only or dual use, and EP results in more reliable QALYs gain as it does not allow e-cigarette initiation among never-smokers. However, over a longer time horizon, the health benefits among later generations of MLA, tax rise and SFG scenarios become apparent and surpass the benefits resulting from policies legalising e-cigarettes.

\section{Combinations of scenarios}

The combination of MLA and moderate tax (TAX5) could perform comparably with aggressive tax (TAX2) in the long term (see figures 3 and 4). Meanwhile, even though EP in combination with MLA could also be as effective as TAX2 in terms of QALY gain, it is projected to lead to a higher prevalence of e/cigarette users. Moreover, allowing ELF along with SFG could enhance or undermine the effect of SFG depending on the level of e-cigarette initiation. In particular, the effect 

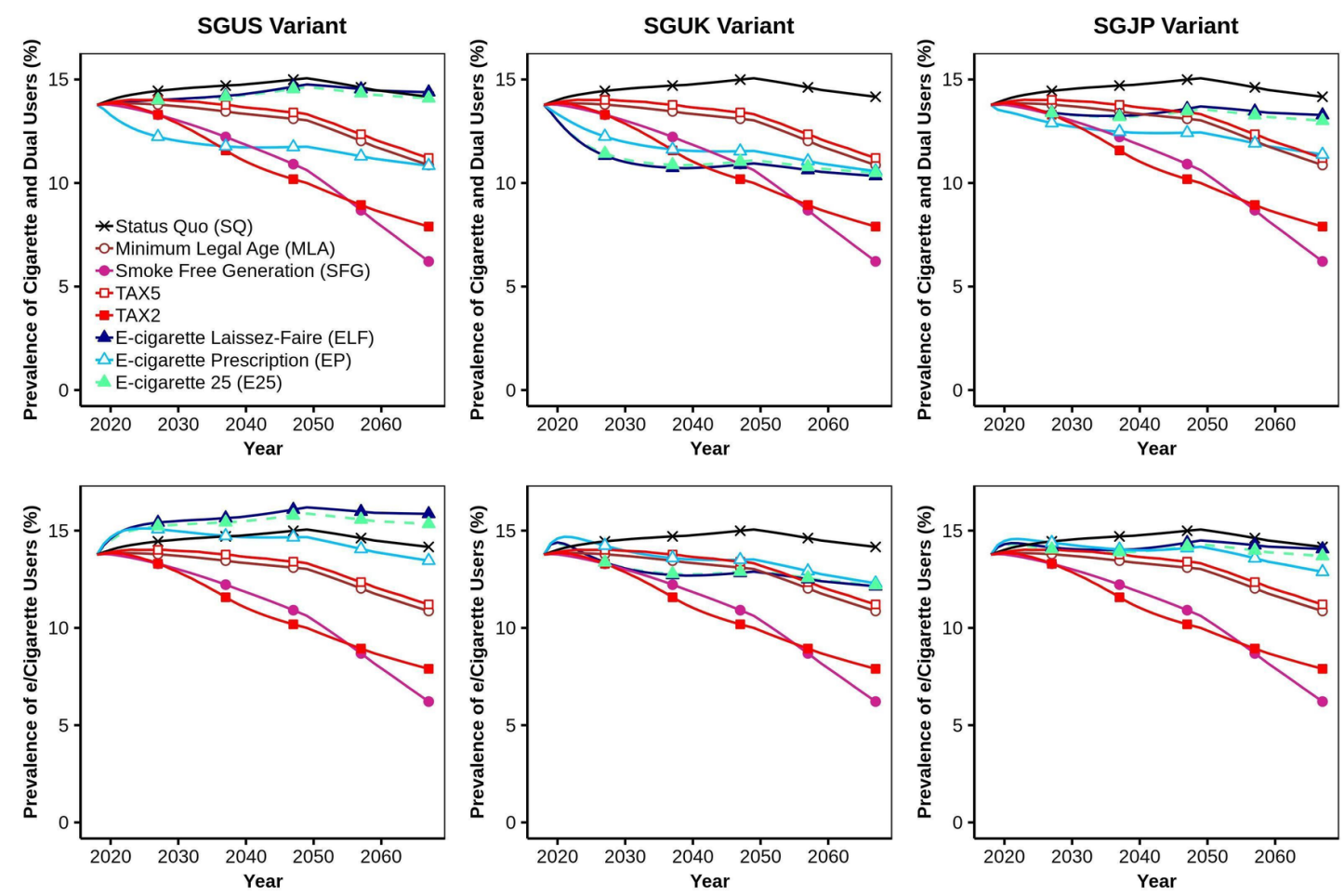

Figure 2 The combined prevalence of cigarette users including dual users and the combined prevalence of cigarette users and e-cigarette users in Singapore in the SGUS variant (left column), SGUK variant (middle column) and SGJP variant (right column) where the transitions between three states of never users, cigarette users, ex-cigarette users in Singapore are, respectively, combined with the transitions to and from e-cigarette users and dual-users in the USA, the UK and Japan under the seven scenarios considered. The MLA scenario involves raising the age at which smoking is legally allowed to 21 years old from 18 years old. The SFG scenario involves prohibiting those born after 2000 from ever smoking. The ELF scenario involves legalising e-cigarettes and allowing anyone aged 18 years or above to use e-cigarettes. The EP scenario allows only smokers to obtain e-cigarettes, under prescriptions from medical doctors. The E25 scenario involves legalising e-cigarettes and allowing only people aged 25 years or above to use ecigarettes. The TAX2 scenario involves raising tax on tobacco consumption once every 2 years for 10 times, each time $10 \%$. The TAX5 scenario involves raising tax on tobacco consumption once every 5 years for 10 times, each time $10 \%$.

of SFG in lowering smoking prevalence and raising QALY are enhanced when featured with ELF in the SGUK variant, where the adoption of e-cigarettes from never smokers is negligible compared with that from current smokers (figure 4, middle column and figure 3 , middle plot), but are undermined in the
SGUS variant where a more considerable proportion of never smokers initiating e-cigarette use (figure 4, left column and figure 3 , left plot).

Tables of estimates of the prevalence of cigarette smokers and QALYs gained are available in online supplement S3 and S4.
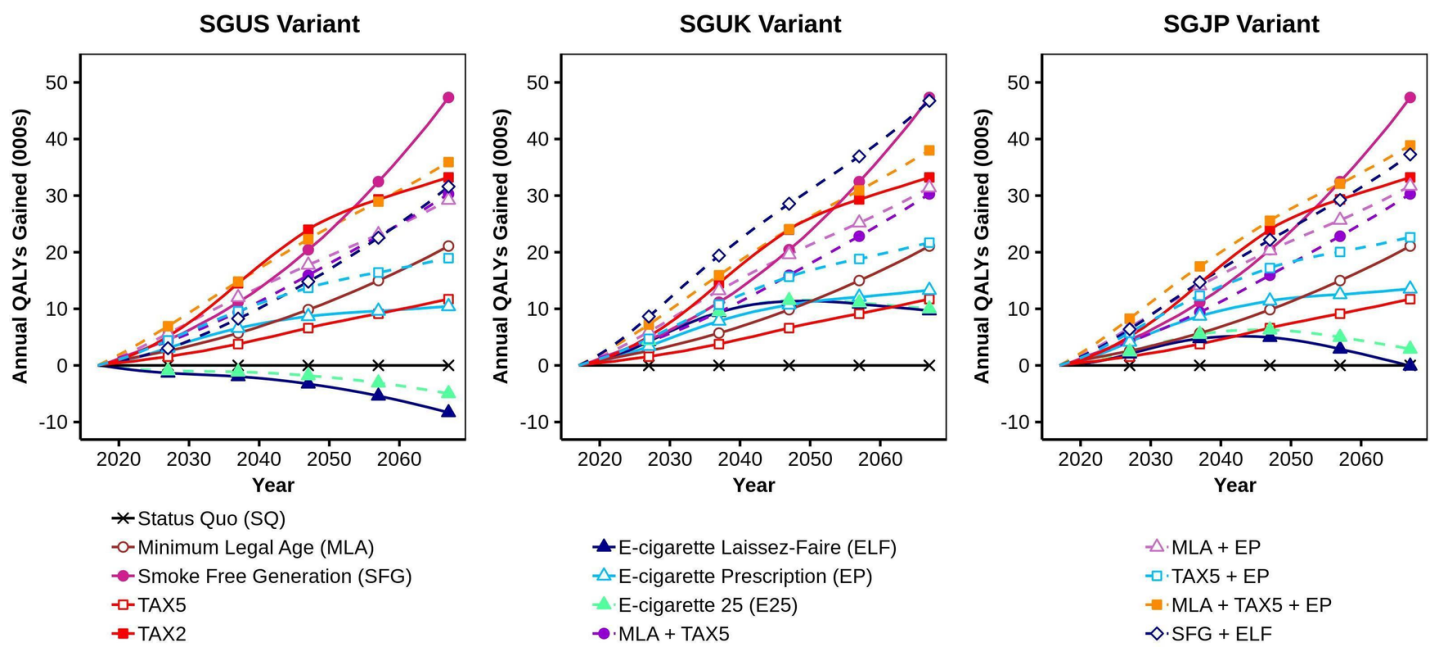

Figure 3 The annual QALY gained in the SGUS variant (left), SGUK variant (middle) and SGJP variant (right) under the seven scenarios of single policies and five scenarios of combined policies as described in the caption of figure 2 and the main text. (Note: the QALY values in this figure are presented without future discounting. Online supplement figure S4-3 in online supplement 4 shows results under $3 \%$ annual discount.). QALYS, quality-adjusted life years. 

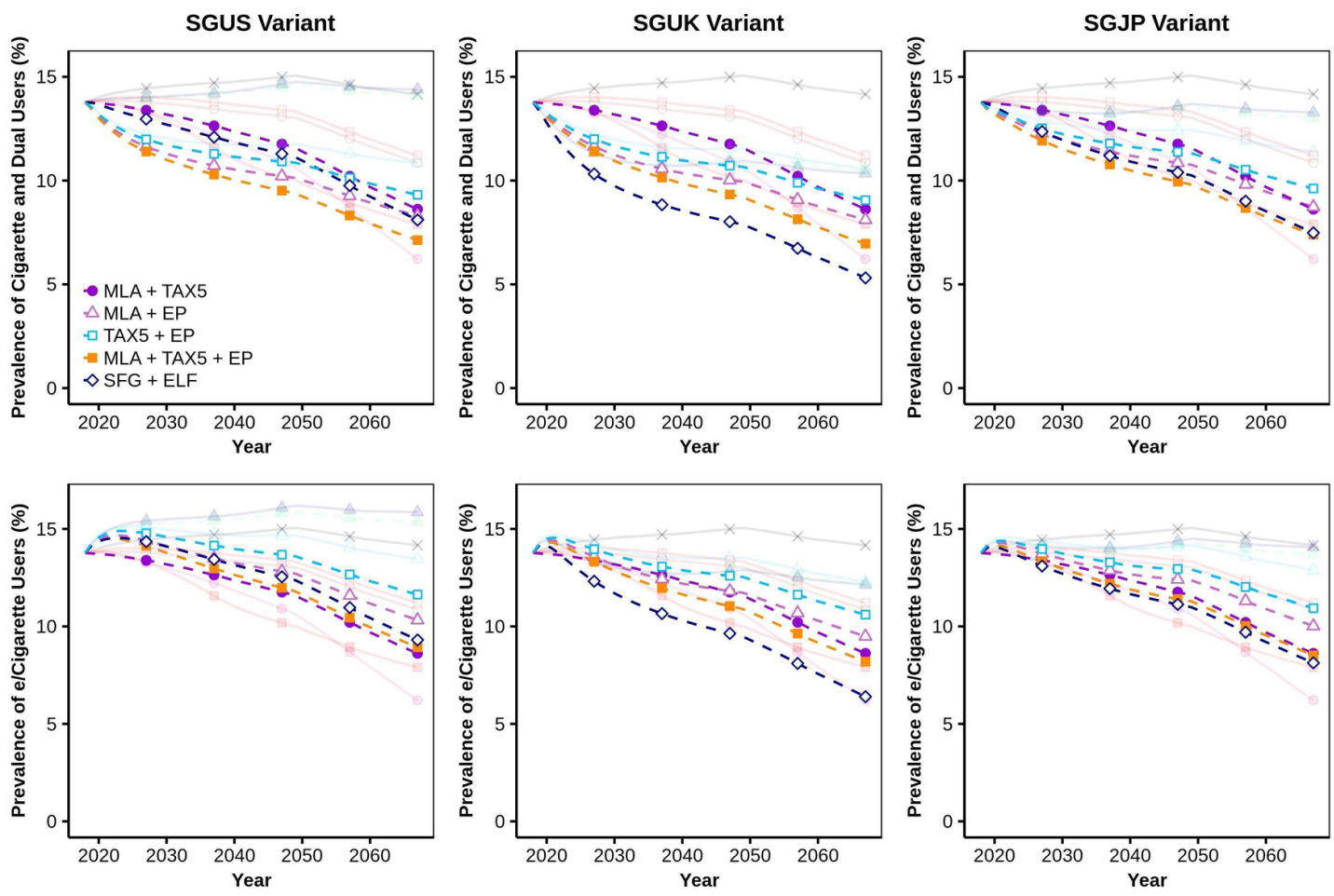

Figure 4 The combined prevalence of cigarette users including dual users and the combined prevalence of cigarette users and e-cigarette users in Singapore in the SGUS variant (left column), SGUK variant (middle column) and SGJP variant (right column) under five combinations of policies: (1) $M L A+T A X 5$, (2) MLA+EP, (3) TAX5+EP, (4) MLA+TAX5+EP, (5) SFG+ELF. ELF, e-cigarette laissez-faire; EP, e-cigarette on prescription; MLA, minimum legal age; $S F G$, smoke-free generation.

\section{Sensitivity analyses}

A full set of sensitivity analyses is presented in online supplement S5 and summarised in box 1 . The estimated health impacts of e-cigarette use are robust to assumptions about the relative risk because, in all three variants, the projected prevalence of e-cigarette use, including dual use, in Singapore is expected to be less than 5\% (see online supplement figure S3-1). Instead, transition probabilities play a more important role in determining the prevalence of cigarette use and hence the QALYs gained in different scenarios. The differences between the SGUS and SGUK results are primarily due to the high rates of transition from conventional to e-cigarettes in the UK, for when we substituted these rates into the SGUS model, the results mirrored those of the SGUK model closely. In addition, when we raised the rates by which current and never smokers adopted e-cigarette use, the overall prevalence of e/cigarette use rose notably. The long-term projection of the results is robust to optimistic assumptions about policies including e-cigarettes, namely, (1) that ex-users of e-cigarettes have the same mortality risk and transition probabilities as never smokers and (2) that no ex-smokers will initiate e-cigarette use.

\section{DISCUSSION}

In this study, we developed models that fused evidence from a country (Singapore) in which e-cigarettes are currently banned with evidence from three countries (Japan, the UK and the USA) in which they now constitute a sizeable share of the market. We observed that the projection of the population's smoking prevalence and health impacts under the ELF scenario is consistent regardless of the foreign country which Singapore was modelled on-there was an initial decline in smoking prevalence as smokers of conventional cigarettes switch to e-cigarettes, which was followed by a plateau as future generations of young adults transition through vaping to smoking.

Our findings are for the most part consistent with those of existing models that investigate policies about e-cigarettes, ${ }^{31} 42-48$ as reviewed in online supplement S6. In particular, the difference between competing models in whether health gains or losses result from introducing e-cigarettes is strongly determined by the ratio of the initiation rates among youth to the adoption rates of e-cigarettes as a cessation aid. Additionally, some former smokers might initiate e-cigarette use, as reflected in the moderate annual rate of transition from ex-smokers to e-cigarette uses in the USA-the rate is 31 per 1000 person-years $(95 \%$ CI 19\%o to 44\%) for age 25-34 years-which is consistent with the rising trend of e-cigarette use among ex-smokers in the UK. ${ }^{49}$

On the other hand, with policies that prohibit or discourage conventional cigarette use, either through raising the age at which cigarettes may be consumed or raising their cost, both reduced smoking prevalence and QALY gain are realised. The policies with the best modelled outcomes were an aggressive tax regime that increases prices every 2 years or the prohibition of smoking among all individuals born after some point in time, that is, the SFG policy. In practice, the impact of tax raise on smoking prevalence could be countered by producers' pricing strategies; ${ }^{50}$ however, its effects on QALYs gained could be enhanced as tax rises also reduce the smoking intensity of current smokers. ${ }^{38}$

Two related policies that emerged from the analysis to be effective are the implementation of the SFG and raising of the MLA. Our results suggest that the MLA policy is a reasonable step, but that the subsequent extension to prohibit the sale of tobacco products to anyone born after the year 2000 would have a noticeably larger long-term effect. Such an extension may also help overcome issues with enforcement of the MLA policy, 
Box 1 Summary of sensitivity analyses (full results may be found in online supplement 5 )

Varying the risk of e-cigarettes

Results appear in online supplement figures S5-1, S5-2.

There are negligible changes in the results, as the main estimate of the proportion of Singapore residents who will adopt e-cigarette only use (E) or dual use (D) and who reach the age when mortality effects are felt most strongly is low $(<5 \%)$ in absolute terms.

\section{Increased adoption of e-cigarettes by never smokers in the SGUK variant}

Results appear in online supplement figure S5-3.

With a low level of e-cigarette initiation by never smokers in the SGUK variant $(0.0 \%-0.4 \%)$, increasing this level of e-cigarette initiation to $0.0 \%-1.2 \%$ does not change the results substantially. However, the accumulation of these small directional changes increases the eventual level of the smoking prevalence in scenarios with e-cigarette on a laissez-faire basis (ie, ELF, E25, SFG+ELF).

\section{Increased adoption of e-cigarettes by current smokers in the SGUS variant}

Results appear in online supplement S5-4.

With more adoption of e-cigarettes by current smokers $(\mathrm{C} \rightarrow \mathrm{E}$ : $0.6 \%-5.1 \%, C \rightarrow D: 0.6 \%-24.9 \%)$, the smoking prevalence in scenarios with e-cigarette on a laissez-faire basis (ie, ELF, E25, $\mathrm{SFG}+\mathrm{ELF}$ ) is lower. However, in comparison with the other scenarios, the smoking prevalence under ELF or E25 is still higher in the long term.

\section{Assuming that neither the proportion of quitters remaining in the ex-smoker compartment nor the proportion of current smokers quitting is affected by the introduction of e-cigarettes \\ Results appear in online supplement S5-5, 55-6 and S5-7. \\ If this assumption were true, the smoking prevalence under the scenarios with e-cigarettes would be lower than the baseline model. Still, in the longer term, the prevalence of e/cigarette users under ELF or E25 is higher than that in other scenarios.}

as it is expected that some adolescents will obtain cigarettes from older peers, thus diluting the benefits of MLA..$^{52}$ Van der Eijk et $a l^{53}$ have shown that SFG is compatible with human rights principles, because although it reduces the liberty of the would-be smokers, this is at a level that is justifiable given the conflicting interests at stake.

Our estimates of transition rates in the USA justify the fear that e-cigarette use might serve as a gateway to conventional cigarette use, with sizeable numbers of e-cigarette users subsequently initiating use of conventional cigarettes (see online supplementary table S2-3 and table S2-4). Our results also highlight the necessity to restrict e-cigarette use, if at all permitted, through requiring prescriptions, or to couple policies using e-cigarettes with SFG to counter the unpredictable developments in e-cigarette initiation. It is also worth emphasising that EP in our model includes face-to-face support to smokers and uses refillable e-cigarettes which can deliver nicotine effectively. EP with limited to no personalised consultation and using e-cigarette cartridges of the first generation has been shown to be less effective. ${ }^{39}$ In countries where aggressive tobacco control policies such as TAX2 or SFG are infeasible, policy makers can consider combinations of less aggressive policies such as MLA plus TAX5 (moderate tax) or MLA plus EP which could offer benefits comparably with TAX2 (aggressive tax) in the long term.

As in all modelling studies, there are many assumptions underpinning the work. The greatest limitation in this study, and for any other modelling study on e-cigarette policy, is the lack of long-term trend data. ${ }^{54}$ In this study, we did not account for changes in transition probabilities over time-except for a gradual decrease in illegal cigarette initiation under SFG, and a slight increase in e-cigarette initiation in the absence of the competing product, cigarettes, in the scenario in which e-cigarettes are made available in tandem with the implementation of the SFG-though changes might be anticipated in particular to e-cigarette use, as vaping becomes normalised. It may be valuable for Singapore's policy makers to revisit this policy question once further data on uptake and risk profiles of e-cigarette use with and without conventional cigarettes become more established.

Furthermore, this policy analysis considered three types of policies (taxation, e-cigarette use and minimum ages), but other policies may also influence the initiation and cessation of nicotine use. Plain or standardised packaging of tobacco products, which results in reduced appeal to consumers, ${ }^{55}$ has been recently proposed by Singapore's Ministry of Health for implementation in the near future, in response to evidences suggesting that such a policy is effective in other high-income societies. ${ }^{556}$ This policy should be evaluated in Singapore to facilitate modelling of its long-term effect and to support (or not) similar policies elsewhere.

In addition, the excess mortality risk due to e-cigarette use is not currently well characterised, so we used a range of 5\%-40\% for this quantity: future studies will hopefully reduce this uncertainty and quantify differences in excess risk by disease. Moreover, the relative mortality risks used for smoking could vary in the future due to the evolving distribution of diseases causing death (eg, should the prevalence of cardiovascular diseases decrease). Besides, we did not calculate the healthcare expenditure associated with diseases caused by smoking. The global economic costs of smoking was estimated to be US\$1.44 trillion in 2012, approximately $1.8 \%$ of the global gross domestic product and $5.7 \%$ of global health expenditure. ${ }^{57}$ Also, we did not account for the effect of smoking or vaping on secondhand smokers, as there was insufficient data to model it. Taking into account additional costs for secondhand smokers would further escalate the costs to both healthcare and economy and preferentially support more aggressive policies.

This modelling study suggests that legalising e-cigarettes may not be the best available approach to tobacco end-game in settings where e-cigarettes are yet to be licensed. In such settings, there is an argument that e-cigarettes, if at all permitted, should be tightly controlled and coupled with policies to restrict uptake of smoking among youths, such as the SFG policy.

Contributors TTTD and KWT contributed to model building, data acquisition, analysis and drafting of the manuscript. ARC contributed to the conception of the model, interpretation of the results and drafting of the manuscript. BSLD, YAL and QY contributed to data acquisition and analysis.

Funding This work was supported by funding from Singapore's Ministry of Health through the Population Health Metrics and Analytics programme and the National Medical Research Council's Centre Grant Programme which funds the Singapore Population Health Improvement Centre (NMRC/CG/C026/2017_NUHS) and by Singapore's National Research Foundation (grant NRF2017VSG-AT3DCM001-022).

Competing interests None declared.

Patient consent for publication Not required.

Provenance and peer review Not commissioned; externally peer reviewed. 
What this paper adds

- We provide an open-cohort model to quantify the public health effects of introducing e-cigarettes into a new market, applicable to countries with limited data on the potential rates of e-cigarette uptake for never smokers and current smokers.

- We evaluate three practical variants of e-cigarette policies in which e-cigarettes sales are either freely driven by the market forces, restricted to smokers with prescriptions from medical doctors or permitted to people from 25 years old only.

- We compare variants of e-cigarette policies with other effective tobacco policies including minimum legal age, smoke-free generation and tax rises on tobacco consumption.

- We present feasible combinations of less aggressive policies such as minimum legal age plus moderate tax or e-cigarette prescription which could be as effective as an aggressive tax policy in the long term.

Data availability statement Data are available in a public, open access repository.

Open access This is an open access article distributed in accordance with the Creative Commons Attribution Non Commercial (CC BY-NC 4.0) license, which permits others to distribute, remix, adapt, build upon this work non-commercially, and license their derivative works on different terms, provided the original work is properly cited, appropriate credit is given, any changes made indicated, and the use is non-commercial. See: http://creativecommons.org/licenses/by-nc/4.0/.

\section{ORCID iD}

Thi Thanh Tra Doan http://orcid.org/0000-0002-1680-288X

\section{REFERENCES}

1 Regan AK, Promoff G, Dube SR, et al. Electronic nicotine delivery systems: adult use and awareness of the 'e-cigarette' in the USA. Tob Control 2013;22:19-23.

2 Goniewicz ML, Gawron M, Nadolska J, et al. Rise in electronic cigarette use among adolescents in Poland. J Adolesc Health 2014;55:713-5.

3 White J, Li J, Newcombe R, et al. Tripling use of electronic cigarettes among New Zealand adolescents between 2012 and 2014. J Adolesc Health 2015;56:522-8.

4 McCarthy M. "Alarming" rise in popularity of e-cigarettes is seen among US teenagers as use triples in a year. BMJ 2015;350:h2083.

5 de Lacy E, Fletcher A, Hewitt G, et al. Cross-Sectional study examining the prevalence, correlates and sequencing of electronic cigarette and tobacco use among 11-16-year olds in schools in Wales. BMJ Open 2017;7:e012784.

6 Pokhrel P, Fagan P, Little MA, et al. Smokers who try e-cigarettes to quit smoking: findings from a multiethnic study in Hawaii. Am J Public Health 2013;103:e57-62.

7 Farrimond $\mathrm{H}, \mathrm{Abraham} \mathrm{C}$. Developing e-cigarette friendly smoking cessation services in England: staff perspectives. Harm Reduct J 2018;15:38.

8 Liu X, Lu W, Liao S, et al. Efficiency and adverse events of electronic cigarettes: a systematic review and meta-analysis (PRISMA-compliant article). Medicine 2018;97:e0324.

9 Barrington-Trimis JL, Urman R, Berhane K, et al. E-Cigarettes and future cigarette use. Pediatrics 2016;138:e20160379.

10 Schneider S, Diehl K. Vaping as a catalyst for smoking? an initial model on the initiation of electronic cigarette use and the transition to tobacco smoking among adolescents. NICTOB 2016;18:647-53.

11 Laverty AA, Filippidis FT, Vardavas CI. Patterns, trends and determinants of e-cigarette use in 28 European Union member states 2014-2017. Prev Med 2018;116:13-18.

12 Institute for Global Tobacco Control, Johns Hopkins Bloomberg School of Public Health. Country laws regulating e-cigarettes: a policy scan. Available: https://www. globaltobaccocontrol.org/e-cigarette_policyscan [Accessed 15 Nov 2018].

13 Lim J, Lee JA, Fong GT, et al. Awareness and use of e-cigarettes and Vaping behaviors among Korean adult smokers: ITC 2016 Korean study. JKSRNT 2018;9:S11-S21.

14 Tabuchi T, Kiyohara K, Hoshino T, et al. Awareness and use of electronic cigarettes and heat-not-burn tobacco products in Japan. Addiction 2016;111:706-13.

15 Kaur J, Rinkoo AV. Getting real with the upcoming challenge of electronic nicotine delivery systems: the way forward for the south-east Asia region. Indian J Public Health 2017;61(Suppl 1):S7-S11.

16 Tobacco control laws. Singapore details. Available: https://www.tobaccocontrollaws. org/legislation/country/singapore/summary [Accessed 4 Sep 2018].
17 van der Eijk Y, Lee JK, M Ling P. How menthol is key to the tobacco industry's strategy of recruiting and retaining young smokers in Singapore. J Adolesc Health 2019;64:347-54.

18 HSA. Prohibition on certain products. Available: https://www.hsa.gov.sg/content/hsa/ en/Health_Products_Regulation/Tobacco_Control/Overview/Tobacco_Legislation/ Prohibition_on_Certain_Products.html [Accessed 16 Jun 2019].

19 Singapore Government. Tobacco (Control of Advertisements and Sale) Act - Singapore Statutes Online. Available: https://sso.agc.gov.sg/Act/TCASA1993 [Accessed 15 Nov 2018].

20 Kobus K. Peers and adolescent smoking. Addiction 2003;98 Suppl 1:37-55.

21 Geckova A, van Dijk JP, van Ittersum-Gritter T, et al. Determinants of adolescents' smoking behaviour: a literature review. Cent Eur J Public Health 2002;10:79-87.

22 Khoo D, Chiam Y, Ng P, et al. Phasing-out tobacco: proposal to deny access to tobacco for those born from 2000. Tob Control 2010;19:355-60.

23 United Nations Population Division. wpp2017: world population prospects 2017, 2018. Available: https://CRAN.R-project.org/package=wpp2017 [Accessed 28 Oct 2018].

$24 \mathrm{MOH}$. Trend in adult mortality in Singapore. Available: https://www.moh.gov.sg/ resources-statistics/information-paper/trend-in-adult-mortality-in-singapore [Accessed 2 Jun 2019].

25 United States National Institute on Drug Abuse (NIDA). Population assessment of tobacco and health (path) series 2016.

26 ONS. Home - Office for National Statistics. Available: https://www.ons.gov.uk/ [Accessed 15 Aug 2018].

27 Tabuchi T, Gallus S, Shinozaki T, et al. Heat-not-burn tobacco product use in Japan: its prevalence, predictors and perceived symptoms from exposure to secondhand heatnot-burn tobacco aerosol. Tob Control 2018;27:e25-33.

28 Lumley T. Analysis of complex survey samples. J Stat Softw 2004;9.

29 Plummer M, Stukalov A, Denwood M. rjags: Bayesian graphical models using MCMC, 2018. Available: https://CRAN.R-project.org/package=rjags [Accessed 11 Oct 2018].

30 Kaufman DW, Helmrich SP, Rosenberg L, et al. Nicotine and carbon monoxide content of cigarette smoke and the risk of myocardial infarction in young men. $N$ Eng/ J Med 1983;308:409-13.

31 Levy DT, Borland R, Lindblom EN, et al. Potential deaths averted in USA by replacing cigarettes with e-cigarettes. Tob Control 2018;27:18-25.

32 RCP London. Nicotine without smoke: tobacco harm reduction, 2016. Available: https://www.rcplondon.ac.uk/projects/outputs/nicotine-without-smoke-tobacco-harmreduction-0 [Accessed 28 Jun 2019].

33 Nutt DJ, Phillips LD, Balfour D, et al. Estimating the harms of nicotine-containing products using the MCDA approach. Eur Addict Res 2014;20:218-25.

34 Glantz SA. Need for examination of broader range of risks when predicting the effects of new tobacco products. NICTOB 2017;19:266-7.

35 Center for Tobacco Control Research and Education. The evidence that e-cigs increase cardiovascular risk keeps piling up: effects on heart rhythm and oxidative stress. Available: https://tobacco.ucsf.edu/evidence-e-cigs-increase-cardiovascularrisk-keeps-piling-effects-heart-rhythm-and-oxidative-stress [Accessed 28 Jun 2019].

36 Thun MJ. Age and the Exposure-Response Relationships Between Cigarette Smoking and Premature Death in Cancer Prevention Study II. In: National cancer Institute, smoking and tobacco control. monograph 8: changes in cigarette related disease risks and their implication for prevention and control, 1997: 383-412.

37 Australian Government Department of Health and Ageing, M. H. and C. D. D. Tobacco taxation. Available: https://www.health.gov.au/internet/main/publishing.nsf/Content/ tobacco-tax [Accessed 28 Jun 2019].

38 World Health Organization. Factor affecting consumer behavior. Available: http:// www.who.int/tobacco/economics/2_1ffactorsaffectingconsumerbehavior.pdf [Accessed 13 Sep 2018].

39 Hajek P, Phillips-Waller A, Przulj D, et al. A randomized trial of e-cigarettes versus Nicotine-Replacement therapy. N Engl J Med 2019;380:629-37.

40 R Core Team. R: a language and environment for statistical computing; 2018

41 yWorks. yEd graph editor 2018.

42 Cherng ST, Tam J, Christine PJ, et al. Modeling the effects of e-cigarettes on smoking behavior: implications for future adult smoking prevalence. Epidemiology 2016;27:819-26

43 Hill A, Camacho OM. A system dynamics modelling approach to assess the impact of launching a new nicotine product on population health outcomes. Regul Toxicol Pharmacol 2017;86:265-78.

44 Kalkhoran S, Glantz SA. Modeling the health effects of expanding e-cigarette sales in the United States and United Kingdom: a Monte Carlo analysis. JAMA Intern Med 2015;175:1671-80.

45 Levy DT, Borland R, Villanti AC, et al. The application of a Decision-Theoretic model to estimate the public health impact of Vaporized nicotine product initiation in the United States. Nicotine Tob Res 2017;19:149-59.

46 Warner KE, Mendez D. E-Cigarettes: comparing the possible risks of increasing smoking initiation with the potential benefits of increasing smoking cessation. Nicotine Tob Res 2019;21:41-7.

47 Soneji SS, Sung H-Y, Primack BA, et al. Quantifying population-level health benefits and harms of e-cigarette use in the United States. PLOS One 2018;13:e0193328. 
48 Petrović-van der Deen FS, Wilson N, Crothers A, et al. Potential country-level health and cost impacts of legalizing domestic sale of Vaporized nicotine products. Epidemiology 2019;30:396-404.

49 Smoking In England. Latest statistics. Available: http://www.smokinginengland.info/ latest-statistics/ [Accessed 21 Nov 2018].

50 Hiscock R, Branston JR, McNeill A, et al. Tobacco industry strategies undermine government Tax policy: evidence from commercial data. Tob Control 2018;27:488-97.

51 Castrucci BC, Gerlach KK, Kaufman NJ, et al. Adolescents' acquisition of cigarettes through noncommercial sources. J Adolesc Health 2002;31:322-6.

52 Richardson L, Hemsing N, Greaves L, et al. Preventing smoking in young people: a systematic review of the impact of access interventions. Int J Environ Res Public Health 2009;6:1485-514.
53 van der Eijk Y, Porter G. Human rights and ethical considerations for a tobacco-free generation. Tob Control 2015;24:238-42.

54 Kalkhoran S, Glantz SA. E-Cigarettes and smoking cessation in real-world and clinical settings: a systematic review and meta-analysis. Lancet Respir Med 2016;4:116-28.

55 Hughes N, Arora M, Grills N. Perceptions and impact of plain packaging of tobacco products in low and middle income countries, middle to upper income countries and low-income settings in high-income countries: a systematic review of the literature. BMJ Open 2016;6:e010391.

56 Smith CN, Kraemer JD, Johnson AC, et al. Plain packaging of cigarettes: do we have sufficient evidence? Risk Manag Healthc Policy 2015;8:21-30.

57 Goodchild M, Nargis N, Tursan d'Espaignet E. Global economic cost of smokingattributable diseases. Tob Control 2018;27:58-64. 